\title{
Evaluating biomechanical properties of murine embryos using Brillouin microscopy and optical coherence tomography
}

Raksha Raghunathan

Jitao Zhang

Chen $\mathrm{Wu}$

Justin Rippy

Manmohan Singh

Kirill V. Larin

Giuliano Scarcelli 


\title{
Evaluating biomechanical properties of murine embryos using Brillouin microscopy and optical coherence tomography
}

\author{
Raksha Raghunathan, ${ }^{a, \dagger}$ Jitao Zhang, ${ }^{\mathrm{b}, \dagger}$ Chen Wu, ${ }^{\mathrm{a}}$ Justin Rippy, ${ }^{\mathrm{a}}$ Manmohan Singh, ${ }^{\mathrm{a}}$ Kirill V. Larin, ${ }^{\mathrm{a}, \mathrm{c}, \star}$ and \\ Giuliano Scarcellib,* \\ aUniversity of Houston, Department of Biomedical Engineering, Houston, Texas, United States \\ bUniversity of Maryland, Fischell Department of Bioengineering, College Park, Maryland, United States \\ 'Tomsk State University, Interdisciplinary Laboratory of Biophotonics, Tomsk, Russia
}

\begin{abstract}
Embryogenesis is regulated by numerous changes in mechanical properties of the cellular microenvironment. Thus, studying embryonic mechanophysiology can provide a more thorough perspective of embryonic development, potentially improving early detection of congenital abnormalities as well as evaluating and developing therapeutic interventions. A number of methods and techniques have been used to study cellular biomechanical properties during embryogenesis. While some of these techniques are invasive or involve the use of external agents, others are compromised in terms of spatial and temporal resolutions. We propose the use of Brillouin microscopy in combination with optical coherence tomography (OCT) to measure stiffness as well as structural changes in a developing embryo. While Brillouin microscopy assesses the changes in stiffness among different organs of the embryo, OCT provides the necessary structural guidance. $\odot 2017$ Society of Photo-Optical Instrumentation Engineers (SPIE) [DOI: 10.1117/1.JBO.22.8.086013]
\end{abstract}

Keywords: Brillouin microscopy; optical coherence tomography; embryonic imaging; tissue mechanics.

Paper 170371R received Jun. 8, 2017; accepted for publication Aug. 3, 2017; published online Aug. 31, 2017.

\section{Introduction}

Mechanical forces play a significant role in the development of different organs. For example, the heart tube, which begins to contract at embryonic day (E) 8.5 , circulates plasma through the vascular system for the first few hours following the first contractions. After the heartbeat strengthens, erythrocytes from blood islands begin to circulate, and, within the next $24 \mathrm{~h}$, blood flow becomes stronger and the heart loops, eventually forming the chambers. Here, the hemodynamic force exerted by the blood flow induces vascular remodeling. ${ }^{1}$ Similarly, a number of other processes, such as cell differentiation, ${ }^{2-4}$ rate of cell proliferation, ${ }^{5-8}$ and tumor progression, ${ }^{9-11}$ are affected by cellular biomechanical properties. Thus, understanding the mechanical changes that occur during development will help us gain a deeper insight into the process of embryogenesis.

Although a number of mechanical parameters affect embryonic development, the morphogenetic flows and tissue deformations that occur as a part of embryonic development are due to two important mechanical factors: the spatial distribution of stresses generated by the cells and the local mechanical properties of the surrounding tissues. ${ }^{12}$ Measuring these mechanical factors is a significant challenge. Several techniques have been proposed to study the different mechanical parameters that affect embryogenesis. However, the techniques that have been developed for embryonic applications have mostly been used to study cellular forces or stresses. ${ }^{13}$ Considerably less in vivo work has been done to measure local material properties of developing tissues.

\footnotetext{
*Address all correspondence to: Kirill V. Larin, E-mail: klarin@uh.edu; Giuliano Scarcelli, E-mail: scarc@umd.edu

†The authors contributed equally.
}

Techniques that have been used in different settings (in vivo and in vitro) to measure the mechanical properties of developing embryos include laser ablation, ${ }^{14}$ tissue dissection and relaxation, ${ }^{15-18}$ force inference, ${ }^{19-21}$ micropipette aspiration, ${ }^{22-25}$ cantilevers and atomic force microscopy (AFM), ${ }^{26-28}$ microindenters and microplates, ${ }^{29-32}$ three-dimensional (3-D) traction force microscopy, ${ }^{33}$ optical tweezers (OT), ${ }^{34}$ magnetic tweezers, ${ }^{35}$ microrheology, ${ }^{36}$ Förster resonance energy transfer-based tension sensors, ${ }^{37}$ and droplet-based sensors (DS). ${ }^{38}$ While these techniques have revealed valuable information about the mechanophysiology of embryonic development, some of them are invasive, involve the use of external agents, or have poor spatial and temporal resolutions. For example, microindentation and AFM require physical contact with the sample, and force inference cannot provide quantitative material properties. In contrast, OT and DS do provide quantitative information but require microinjections of external agents or involve complex calibrations.

In the past, elastography has also been used to study mechanical changes that occur during embryonic development. The techniques used include AFM elastography, ${ }^{39}$ optical coherence elastography (OCE) ${ }^{30}$ and acoustic radiation force impulse (ARFI) imaging. ${ }^{40}$ However, resolution constraints for subcellular imaging with OCE and ARFI and the need for external loading to induce deformations might have detrimental effects on the developing embryo or may cause external perturbations to the already existing mechanical forces.

Brillouin microscopy has recently shown particular promise for tissue and cell biomechanical applications. ${ }^{41,42}$ Brillouin microscopy is an all-optical method that probes the mechanical properties of material via light scattering and thus is noncontact

$1083-3668 / 2017 / \$ 25.00$ @ 2017 SPIE 
and noninvasive. In addition, it does not need to deform the sample with an external load during measurement and can achieve submicron resolution when using a high numericalaperture (NA) objective lens. Brillouin microscopy has demonstrated its use in various biological applications, such as the characterization of crystalline lens and cornea of the eye, ${ }^{43-45}$ the measurement of fibrous proteins of extracellular matrix, ${ }^{46}$ and the mapping of cellular and subcellular mechanics with live cells. ${ }^{47-50}$

Optical coherence tomography (OCT) ${ }^{51}$ is a well-established optical imaging modality that is capable of live 3-D imaging of embryos with high spatial and temporal resolution. ${ }^{52}$ Due to its ability to provide cross-sectional images of a specimen noninvasively with high resolution, OCT has become a powerful tool in developmental biology. ${ }^{52}$

In this pilot study, we use Brillouin microscopy in combination with structural imaging provided by OCT (Br-OCT) to analyze the spatial stiffness distribution of a developing embryo. Due to the micrometer-scale resolutions of both techniques, we generated two-dimensional (2-D) elasticity maps of a murine E8.5 embryo using Brillouin microscopy and correlated these results with structural images obtained with OCT. Br-OCT provided a completely noninvasive and noncontact method for imaging the structure and assessing stiffness changes of an embryo without the use of any external agents. Quantitative results were obtained in terms of Brillouin frequency shift, a measure of stiffness of the tissue.

\section{Methods}

\subsection{Animal Manipulations}

Timed matings of CD-1 mice were set up overnight. The mice were checked for a vaginal plug every morning. The morning when a plug was found was considered E0.5. On E8.5, the embryos were dissected out and were first imaged using a home-built swept source OCT system. The embryos were then kept in $0.9 \%$ saline and transported to the University of Maryland on ice and imaged with Brillouin microscopy within $8 \mathrm{~h}(n=5)$. The embryos were kept cold but not frozen during transportation and were brought back to room temperature for imaging.

\subsection{Brillouin Microscopy}

The Brillouin microscope is built by coupling a Brillouin spectrometer with a confocal microscope, and the setup is shown in Fig. 1. The laser source is a single-mode linearly polarized

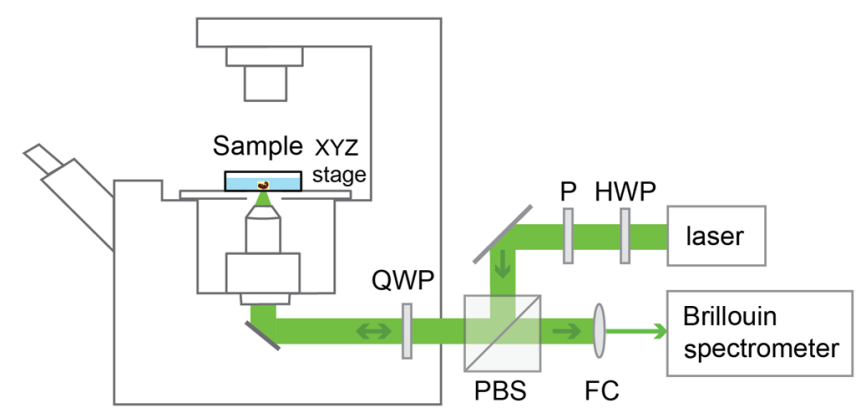

Fig. 1 Sketch of Brillouin microscopy. HWP, half-wave plate; P, polarizer; PBS, polarized beam splitter; QWP, quarter-wave plate; and FC, fiber coupler. 532-nm continuous wave laser (Torus, Laser Quantum), and $\sim 10-\mathrm{mW}$ power was used in the experiment. After passing through a half-wave plate (HWP) and a polarizer (P), the polarization of the laser beam was aligned to the input polarization of the polarized beam splitter (PBS). The beam was then focused into the sample by an objective lens $(\mathrm{NA}=0.6$, magnification $40 \times$ ) with a spot size of $\sim 0.5 \mu \mathrm{m} \times \sim 0.5 \mu \mathrm{m}$ (transverse) by $\sim 2 \mu \mathrm{m}$ (axial). The backward scattering light was collected by the same objective lens and coupled into a Brillouin spectrometer through a fiber coupler (FC). A combination of a quarter-wave plate (QWP) and a PBS was used to ensure that all the scattered light collected by the objective lens was delivered into the spectrometer. The Brillouin spectrometer consisted of a twostage virtually imaged phased array in cross-axis configuration, similar to previous reports. ${ }^{47,53}$ Before and after the measurement, the Brillouin spectrum was calibrated by standard materials with known Brillouin frequency shift. The embryo sample was placed onto a glass bottom dish and surrounded by medium. Brillouin images were acquired by scanning the sample with the help of an automatic XYZ stage. The step size of the scanning was $5 \mu \mathrm{m}$.

\subsection{Optical Coherence Tomography System}

The home-built OCT system consisted of a swept source laser (HSL2000, Santec USA, Corp., Hackensack, New Jersey) with a central wavelength of $\sim 1310 \mathrm{~nm}$, scan range of $\sim 150 \mathrm{~nm}$, A-scan rate of $\sim 30 \mathrm{kHz}$, output power of $\sim 39 \mathrm{~mW}$, and axial resolution of $\sim 11 \mu \mathrm{m}$ (in air). ${ }^{54,55}$ Figure 2 shows a schematic of the OCT system. A-scan averaging was performed to reduce the background noise and enhance signal-to-noise ratio (SNR); five A-scans were taken at each position and then averaged. The images were corrected to physical dimensions assuming that the refractive indices of saline and the embryos were 1.38 .

\subsection{Relationship between Brillouin Frequency Shift and Elastic Moduli}

Spontaneous Brillouin light scattering arises from the interaction of incoming laser light with acoustic phonons inside the material. Within this process, the scattered light will undergo a frequency shift $v_{B}$, which is related to the high-frequency longitudinal modulus $M^{\prime}$ (the ratio of uniaxial stress to uniaxial strain) of the material as

$v_{B}=\frac{2 n}{\lambda} \sqrt{\frac{M^{\prime}}{\rho}} \sin (\theta / 2)$,

where $\lambda$ is the wavelength of the light source, $n$ is the refractive index of the material, $\rho$ is the density of the material, and $\theta$ is the angle between the incident and scattered lights. For backward scattering geometry used in this work, $\theta=180 \mathrm{deg}$. We need to know the factor $\rho / n^{2}$ to extract the longitudinal modulus from the measured Brillouin shift, according to Eq. (1). Although the density and refractive index are probably inhomogeneous at different parts of the embryo, previous studies show that the variation of the ratio $\rho / n^{2}$ is fairly small for various biological samples, such as cornea and cells. ${ }^{43,47}$ This indicates that the measured Brillouin shift is approximately proportional to the standard longitudinal modulus. The relationship between high-frequency longitudinal modulus and traditional quasistatic Young's or shear moduli that are generally used for material 


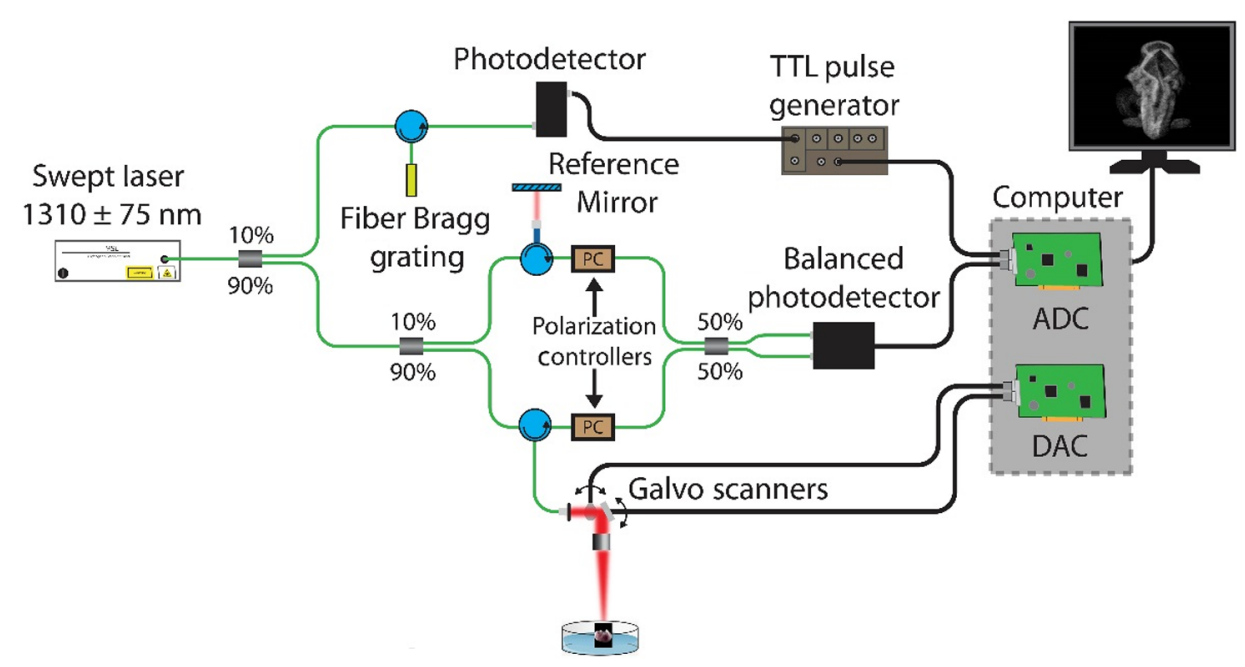

Fig. 2 OCT system schematic. ADC, analog-to-digital converter and DAC, digital-to-analog converter.

characterization is straightforward in crystalline material but not fully understood in biological tissue. For soft matter, such as biopolymers, biological tissue, and cells, the longitudinal modulus is generally much higher than traditional moduli due to the incompressibility of the material and the frequency dependence of the modulus. Empirically, we previously found that the longitudinal modulus, $M^{\prime}$, measured by Brillouin microscopy, has a $\log -\log$ linear relationship to conventional Young's modulus, $E^{\prime}$, through

$\log \left(M^{\prime}\right)=\mathrm{a} \cdot \log \left(E^{\prime}\right)+b$,

where $a$ and $b$ are the material-dependent coefficients. ${ }^{44}$ In this work, we report the Brillouin shift as a metric of mechanical properties of the embryo as it is the direct parameter measured in the experiment.

\section{Results and Discussion}

Multiple embryos were imaged successfully $(n=5)$. Figure 3(a) shows a representative 2-D mechanical map of a sagittal plane of an embryo as measured by Brillouin microscopy. Figure 3(b) shows a similar 2-D sagittal section obtained from the 3-D OCT structural image [Fig. 3(c)]. Using Fig. 3(b) as a reference, the neural folds, developing heart, and closed neural tube/ somites of the embryo in Fig. 3(a) are well-identified.
The mean Brillouin shift values from the different regions labeled in Fig. 4(a) were averaged and are plotted in Fig. 4(b) to show the difference in Brillouin shift between organs of the developing embryo. Based on the linear log-log relationship between the elastic modulus measured by Brillouin microscopy and the conventional Young's modulus in Eq. (2), we observe that the neural tube/somites at this stage (E8.5) are much stiffer compared to the developing brain (neural folds) and the developing heart.

The results depicted here are from one cross section of the embryo. In our future studies, we will image different planes and then calculate the stiffness differences between organs and the changes in stiffness of each organ over time.

One limitation of the current work is that the embryos were dissected out of the yolk sac. The yolk sac is highly scattering and thus requires further development of the Brillouin system to obtain accurate measurements with sufficient SNR. Our future work will involve analyzing the biomechanical properties of developing embryos within the yolk sac because this will allow for longitudinal analysis of embryonic biomechanical properties in live animals.

Figure 5 shows different orientations of 3-D OCT image of the same embryo. It can be clearly seen that the embryo was imaged during neural tube closure. ${ }^{56}$ The neural groove fusion had begun, and we noticed that the rostral/cranial and caudal neuropores were still open. Imaging the embryonic neural

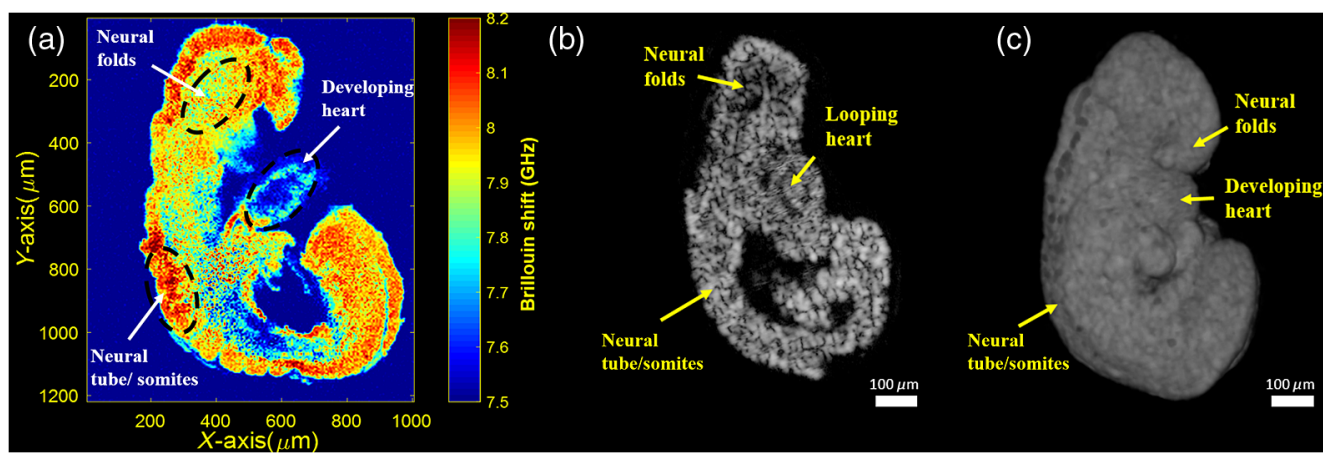

Fig. 3 E8.5 Br-OCT images. (a) 2-D elasticity map obtained using Brillouin microscopy from one sagittal plane, (b) 2-D OCT image of a similar sagittal plane of the same embryo in (a), and (c) 3-D OCT image. 

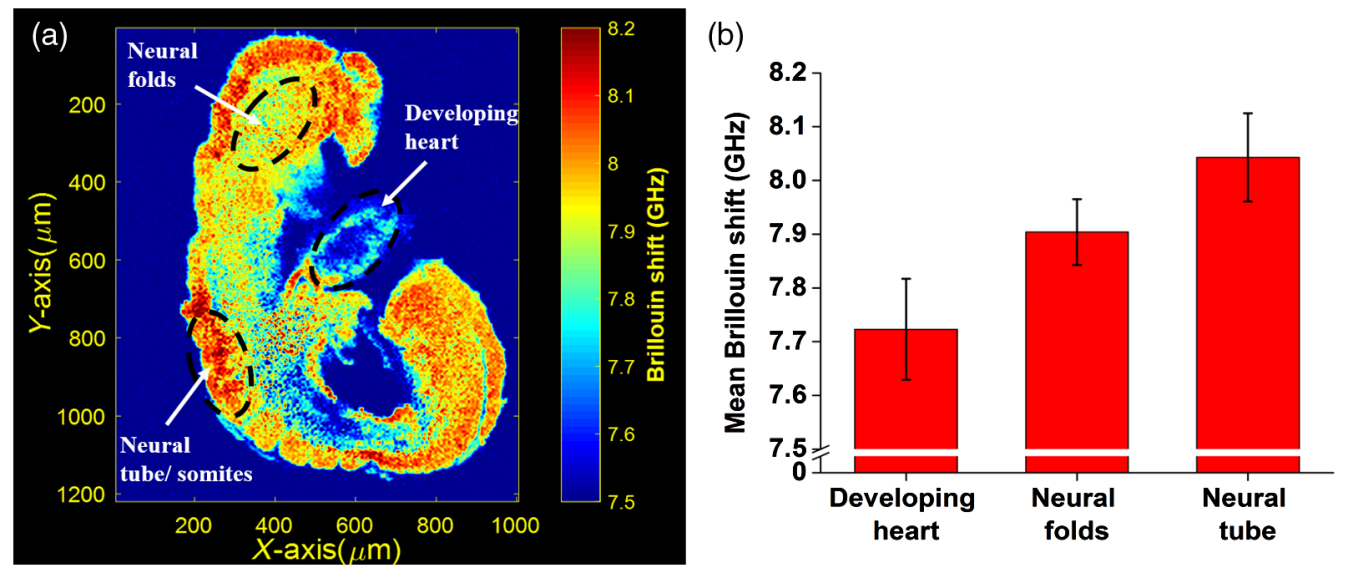

Fig. 4 (a) 2-D elasticity map obtained using Brillouin microscopy from one sagittal plane with labeled regions for quantifying embryo tissue stiffness and (b) stiffness quantifications of labeled regions.

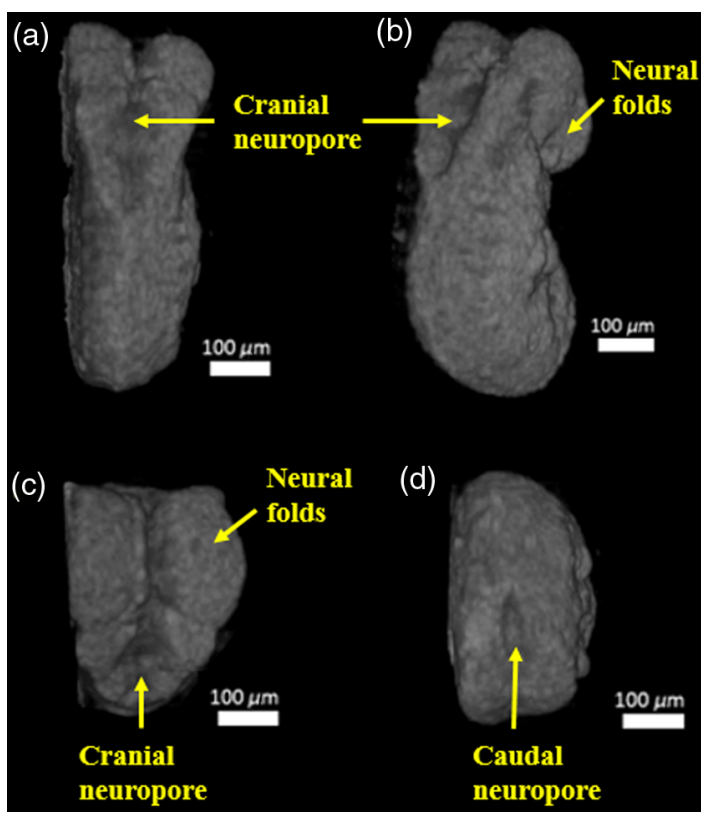

Fig. 5 (a) Dorsal view of the E8.5 embryo, (b) partially rotated dorsal, (c) top-down view of the embryo showing the open cranial neuropore, and (d) bottom-up view of the embryo showing the open caudal neuropore.

tube is crucial because neural tube defects (NTDs) are known to occur when neural tube closure is disrupted. ${ }^{57}$ Although the etiology of NTDs is known to be complex, several genetic as well as environmental factors are known to have an influence on neural tube closure. ${ }^{57}$ However, the mechanisms by which these environmental factors affect neural tube closure are not well-known. Since NTDs present themselves as one of many distinct morphological changes in the embryo, studying the mechanical properties of the cells that are involved in neural tube closure might be useful in understanding the mechanisms causing NTDs. This will be the focus of our future work. By imaging different regions of the neural tube at different developmental stages of the murine embryo, we hope to study how changes in mechanical properties of cells influence NTDs. Since OCT has already been used for imaging and analyzing the closure of a live murine embryonic neural tube, ${ }^{58}$ our future work will entail imaging this process with $\mathrm{Br}-\mathrm{OCT}$ and understanding the influence of teratogens and other environmental factors on neural tube closure.

\section{Conclusion}

This work is a pilot study that assessed stiffness distribution via Brillouin shift in a developing embryo using Br-OCT. A 2-D elasticity map of a sagittal plane of an E8.5 embryo was obtained using Brillouin microscopy, and structural guidance to identify the developing organs was provided by OCT, separately. The results show that the closed neural tube/somites area was stiffer than the developing heart and neural folds (developing brain) at this gestational stage. Our work opens up an area of multimodal imaging for understanding changes in local biomechanical properties and corresponding structural changes with Br-OCT.

\section{Disclosures}

The authors declare that there are no conflicts of interest related to this article.

\section{Acknowledgments}

This work was supported, in part, by grants from the National Institute of Health Nos. R01HL120140 and R01HD086765.

\section{References}

1. J. L. Lucitti et al., "Vascular remodeling of the mouse yolk sac requires hemodynamic force," Development 134(18), 3317-3326 (2007).

2. F. M. Watt and W. T. Huck, "Role of the extracellular matrix in regulating stem cell fate," Nat. Rev. Mol. Cell Biol. 14(8), 467-473 (2013).

3. D. E. Discher, D. J. Mooney, and P. W. Zandstra, "Growth factors, matrices, and forces combine and control stem cells," Science 324(5935), 1673-1677 (2009).

4. A. J. Engler et al., "Matrix elasticity directs stem cell lineage specification," Cell 126(4), 677-689 (2006).

5. S. J. Streichan et al., "Spatial constraints control cell proliferation in tissues," Proc. Natl. Acad. Sci. U. S. A. 111(15), 5586-5591 (2014).

6. C. M. Nelson et al., "Emergent patterns of growth controlled by multicellular form and mechanics," Proc. Natl. Acad. Sci. U. S. A. 102(33), 11594-11599 (2005).

7. M. E. Chicurel, C. S. Chen, and D. E. Ingber, "Cellular control lies in the balance of forces," Curr. Opin. Cell Biol. 10(2), 232-239 (1998).

8. C. S. Chen et al., "Geometric control of cell life and death," Science 276(5317), 1425-1428 (1997). 
9. D. Wirtz, K. Konstantopoulos, and P. C. Searson, "The physics of cancer: the role of physical interactions and mechanical forces in metastasis," Nat. Rev. Cancer 11(7), 512-522 (2011).

10. D. T. Butcher, T. Alliston, and V. M. Weaver, "A tense situation: forcing tumour progression," Nat. Rev. Cancer 9(2), 108-122 (2009).

11. M. J. Paszek et al., "Tensional homeostasis and the malignant phenotype," Cancer Cell 8(3), 241-254 (2005).

12. O. Campas, "A toolbox to explore the mechanics of living embryonic tissues," Semin. Cell. Dev. Biol. 55, 119-130 (2016).

13. K. Sugimura, P.-F. Lenne, and F. Graner, "Measuring forces and stresses in situ in living tissues," Development 143(2), 186-196 (2016).

14. T. Zulueta-Coarasa and R. Fernandez-Gonzalez, "Laser ablation to investigate cell and tissue mechanics in vivo," in Integrative Mechanobiology: Micro- and Nano Techniques in Cell Mechanobiology, C. A. Simmons, D.-H. Kim, and Y. Sun, Eds., pp. 110-127, Cambridge University Press, Cambridge (2015).

15. L. V. Beloussov, J. G. Dorfman, and V. G. Cherdantzev, "Mechanical stresses and morphological patterns in amphibian embryos," J. Embryol. Exp. Morphol. 34(3), 559-574 (1975).

16. A. R. Moore and A. S. Burt, "On the locus and nature of the forces causing gastrulation in the embryos of Dendraster excentricus," J. Exp. Zool. 82(1), 159-171 (1939).

17. R. David et al., "Tissue cohesion and the mechanics of cell rearrangement," Development 141(19), 3672-3682 (2014).

18. O. Luu et al., "Large-scale mechanical properties of Xenopus embryonic epithelium," Proc. Natl. Acad. Sci. U. S. A. 108(10), 4000-4005 (2011).

19. S. Ishihara and K. Sugimura, "Bayesian inference of force dynamics during morphogenesis," J. Theor. Biol. 313, 201-211 (2012).

20. K. K. Chiou, L. Hufnagel, and B. I. Shraiman, "Mechanical stress inference for two dimensional cell arrays," PLoS Comput. Biol. 8(5), e1002512 (2012).

21. K. Sugimura and S. Ishihara, "The mechanical anisotropy in a tissue promotes ordering in hexagonal cell packing," Development 140(19), 4091-4101 (2013).

22. A. Chaigne et al., "A soft cortex is essential for asymmetric spindle positioning in mouse oocytes," Nat. Cell Biol. 15(8), 958-966 (2013).

23. J. L. Maitre et al., "Pulsatile cell-autonomous contractility drives compaction in the mouse embryo," Nat. Cell Biol. 17(7), 849-855 (2015).

24. J. Wen et al., "Automated micro-aspiration of mouse embryo limb bud tissue," in IEEE Int. Conf. on Robotics and Automation, pp. 2667-2672 (2015).

25. M. von Dassow, J. A. Strother, and L. A. Davidson, "Surprisingly simple mechanical behavior of a complex embryonic tissue," PLoS One $\mathbf{5}(12)$, e15359 (2010).

26. Y. Hara et al., "Directional migration of leading-edge mesoderm generates physical forces: implication in Xenopus notochord formation during gastrulation," Dev. Biol. 382(2), 482-495 (2013).

27. R. Feroze et al., "Mechanics of blastopore closure during amphibian gastrulation," Dev. Biol. 398(1), 57-67 (2015).

28. M. Krieg et al., "Tensile forces govern germ-layer organization in zebrafish," Nat. Cell Biol. 10(4), 429-436 (2008).

29. E. A. Zamir and L. A. Taber, "Material properties and residual stress in the stage 12 chick heart during cardiac looping," J. Biomech. Eng. 126(6), 823-830 (2004).

30. B. A. Filas, G. Xu, and L. A. Taber, "Probing regional mechanical properties of embryonic tissue using microindentation and optical coherence tomography," in Tissue Morphogenesis: Methods and Protocols, C. M. Nelson, Ed., pp. 3-16, Springer, New York (2015).

31. E. Farge, "Mechanical induction of twist in the Drosophila foregut/ stomodeal primordium," Curr. Biol. 13(16), 1365-1377 (2003).

32. N. Desprat et al., "Tissue deformation modulates twist expression to determine anterior midgut differentiation in Drosophila embryos," Dev. Cell 15(3), 470-477 (2008).

33. J. Zhou et al., "Force production and mechanical accommodation during convergent extension," Development 142(4), 692-701 (2015).

34. K. Bambardekar et al., "Direct laser manipulation reveals the mechanics of cell contacts in vivo," Proc. Natl. Acad. Sci. U. S. A. 112(5), 14161421 (2015).

35. G. F. Weber, M. A. Bjerke, and D. W. DeSimone, "A mechanoresponsive cadherin-keratin complex directs polarized protrusive behavior and collective cell migration," Dev. Cell 22(1), 104-115 (2012).
36. A. D. Wessel et al., "The mechanical properties of early Drosophila embryos measured by high-speed video microrheology," Biophys. $J$. 108(8), 1899-1907 (2015).

37. D. Cai et al., "Mechanical feedback through E-cadherin promotes direction sensing during collective cell migration," Cell 157(5), 1146-1159 (2014).

38. O. Campas et al., "Quantifying cell-generated mechanical forces within living embryonic tissues," Nat. Methods 11(2), 183-189 (2014).

39. N. R. Chevalier et al., "Measuring the micromechanical properties of embryonic tissues," Methods 94, 120-128 (2016).

40. J. Park et al., "Acoustic radiation force impulse (ARFI) imaging of zebrafish embryo by high-frequency coded excitation sequence," Ann. Biomed. Eng. 40(4), 907-915 (2012).

41. G. Scarcelli and S. H. Yun, "Confocal Brillouin microscopy for three-dimensional mechanical imaging," Nat. Photonics 2(1), 39-43 (2008).

42. B. F. Kennedy, P. Wijesinghe, and D. D. Sampson, "The emergence of optical elastography in biomedicine," Nat. Photonics 11(4), 215-221 (2017).

43. G. Scarcelli, R. Pineda, and S. H. Yun, "Brillouin optical microscopy for corneal biomechanics," Invest. Ophthalmol. Vis. Sci. 53(1), 185-190 (2012).

44. G. Scarcelli, P. Kim, and S. H. Yun, "In vivo measurement of age-related stiffening in the crystalline lens by Brillouin optical microscopy," Biophys. J. 101(6), 1539-1545 (2011).

45. G. Scarcelli and S. H. Yun, "In vivo Brillouin optical microscopy of the human eye," Opt. Express 20(8), 9197-9202 (2012).

46. F. Palombo et al., "Biomechanics of fibrous proteins of the extracellular matrix studied by Brillouin scattering," J. R. Soc. Interface 11(101), 20140739 (2014).

47. G. Scarcelli et al., "Noncontact three-dimensional mapping of intracellular hydromechanical properties by Brillouin microscopy," Nat. Methods 12(12), 1132-1134 (2015).

48. G. Antonacci and S. Braakman, "Biomechanics of subcellular structures by non-invasive Brillouin microscopy," Sci. Rep. 6, 37217 (2016).

49. K. Elsayad et al., "Mapping the subcellular mechanical properties of live cells in tissues with fluorescence emission-Brillouin imaging," Sci. Signal 9(435), rs5 (2016).

50. J. Zhang et al., "Brillouin flow cytometry for label-free mechanical phenotyping of the nucleus," Lab Chip 17(4), 663-670 (2017).

51. D. Huang et al., "Optical coherence tomography," Science 254(5035), 1178-1181 (1991).

52. R. Raghunathan et al., "Optical coherence tomography for embryonic imaging: a review," J. Biomed. Opt. 21(5), 050902 (2016).

53. G. Scarcelli and S. H. Yun, "Multistage VIPA etalons for high-extinction parallel Brillouin spectroscopy," Opt. Express 19(11), 1091310922 (2011).

54. R. Manapuram, V. Manne, and K. Larin, "Development of phasestabilized swept-source OCT for the ultrasensitive quantification of microbubbles," Laser Phys. 18(9), 1080-1086 (2008).

55. R. K. Manapuram, V. G. R. Manne, and K. V. Larin, "Phase-sensitive swept source optical coherence tomography for imaging and quantifying of microbubbles in clear and scattering media," J. Appl. Phys. 105(10), 102040 (2009).

56. C. Pyrgaki et al., "Dynamic imaging of mammalian neural tube closure," Dev. Biol. 344(2), 941-947 (2010).

57. J. B. Wallingford et al., "The continuing challenge of understanding, preventing, and treating neural tube defects," Science 339(6123), 1222002 (2013).

58. S. Wang et al., "Dynamic imaging and quantitative analysis of cranial neural tube closure in the mouse embryo using optical coherence tomography," Biomed. Opt. Express 8(1), 407-419 (2017).

Raksha Raghunathan received her undergraduate engineering degree in electronics and communication engineering from Anna University, India, in 2013. Currently, she is a part of the Biomedical Optics Laboratory, and she is pursuing her $\mathrm{PhD}$ in biomedical engineering at the University of Houston. Her research interests include understanding the process of embryonic development and the effects of different teratogens on it using noninvasive imaging methods.

Jitao Zhang is a postdoc in the Bioengineering Department, the University of Maryland, College Park. He received his BS degree 
and $\mathrm{PhD}$ in optical engineering from Wuhan University and Tsinghua University in 2006 and 2010, respectively. His current interests include development and application of innovative Brillouin techniques. He has also worked in other optical fields, such as terahertz spectroscopy, ellipsometry, and optical interferometry.

Chen Wu received his BS degree in optical information science and technology from the Ocean University of China in 2010 and his MS degree in biomedical engineering from Martin Luther University HalleWittenberg, Germany, in 2013. Currently, he is a PhD candidate in the Department of Biomedical Engineering, the University of Houston. His research interests are in the development of optical coherence tomography technique and the application in different biomedical fields, such embryonic imaging, ophthalmology, and tissue biomechanics.

Justin Rippy received his BSc degree in chemistry and cell biology from the University of Mary Hardin-Baylor in 2012. Currently, he is pursuing his $\mathrm{PhD}$ in the Department of Biomedical Engineering, the University of Houston. He has been with Dr. Kirill Larin's Biomedical Optics Laboratory since the fall of 2016. His research interests include the development of biomedical imaging modalities, the application of optical imaging for embryology, and tissue mechanical contrast detection using medical imaging.
Manmohan Singh received his BS degree in biomedical engineering from the University of Houston in 2014 and is currently pursuing his $\mathrm{PhD}$ in the same department. Since the fall of 2010 , he has been with Dr. Kirill Larin's Biomedical Optics Laboratory. His research interests include utilizing biomedical imaging for the detection and monitoring of diseases and utilizing and developing elastographic methods for investigating the biomechanical properties of tissues.

Kirill $\mathbf{V}$. Larin is a professor of biomedical engineering at the University of Houston. He received his MS degree in laser physics and mathematics from the Saratov State University in 1995 and his $\mathrm{PhD}$ in biomedical engineering from the University of Texas Medical Branch, Galveston, in 2002. He has published more than 100 papers in the field of biomedical optics and biophotonics. He was inducted as a fellow of SPIE in 2015 and OSA in 2016.

Giuliano Scarcelli is an assistant professor of bioengineering at the University of Maryland. He received his $\mathrm{PhD}$ in physics from the University of Maryland, Baltimore County, in 2006. Before joining Maryland, he was at the Wellman Center for Photomedicine, Harvard Medical School, for eight years, first as a postdoc, then an instructor, and an assistant professor. He has been the recipient of the Human Frontier Science Program Young Investigator Award and the National Institutes of Health Quantitative Career Award. 